\title{
Estado del arte en robots de asistencia en hospitales, en entornos infecciosos (COVID-19)
}

\author{
Amparo Tirado-Bou, Raúl Marín-Prades, Pedro J. Sanz, José V. Martí \\ Universitat Jaume I de Castellón \\ al028901, rmarin, sanzp, vmarti at uji.es
}

\begin{abstract}
Resumen
En el presente artículo mostramos el estado del arte de los sistemas robóticos que están siendo desarrollados (algunos de los cuales ya en uso), para trabajar en entornos infecciosos, por ejemplo áreas de riesgo asociadas a COVID-19. Los sistemas permiten que el personal sanitario trabaje con mayor seguridad, e incrementan la seguridad también de los propios pacientes, permitiendo el seguimiento de los mismos, así como la desinfección de áreas expuestas al virus, entre otras potenciales aplicaciones. El trabajo futuro, mostrará el resultado de entrevistas con personal sanitario, los cuales han presentado requisitos para un potencial futuro sistema robótico asistencial en este ámbito.
\end{abstract}

Palabras clave: COVID-19, Robótica Sanitaria, Desinfección, Sensores

\section{INTRODUCCIÓN}

Durante la pandemia del COVID-19, se ha evidenciado la necesidad de minimizar el contacto físico, sin perder la atención y el cuidado que los pacientes merecen. Por todo ello se ha presentado la necesidad de desarrollar dispositivos robóticos que permitan contribuir a estas tareas.

El personal sanitario han sido sobre-expuesto al virus, lo que ha puesto en peligro el sistema sanitario mundial.

El trabajo remoto se ha convertido en un aliado en la lucha contra el virus, pero en los entornos donde este no es posible, el número de contagios ha crecido de forma exponencial. En estos casos, los sistemas de protección personal (PPE) se han vuelto imprescindibles.

Hemos de recordar que el protocolo que han de seguir el personal sanitario previo a ingresar en contacto con pacientes con positivo para "COVID19", supone el consumo de equipos de protección personal desechables, como podemos ver en el diagrama mostrado en la figura 1, estos suponen un coste económico importante y también un coste temporal que en muchos casos puede ser evitable $^{1}$.

\section{Pasos para quitarse el equipo de protección personal, incluida la bata}

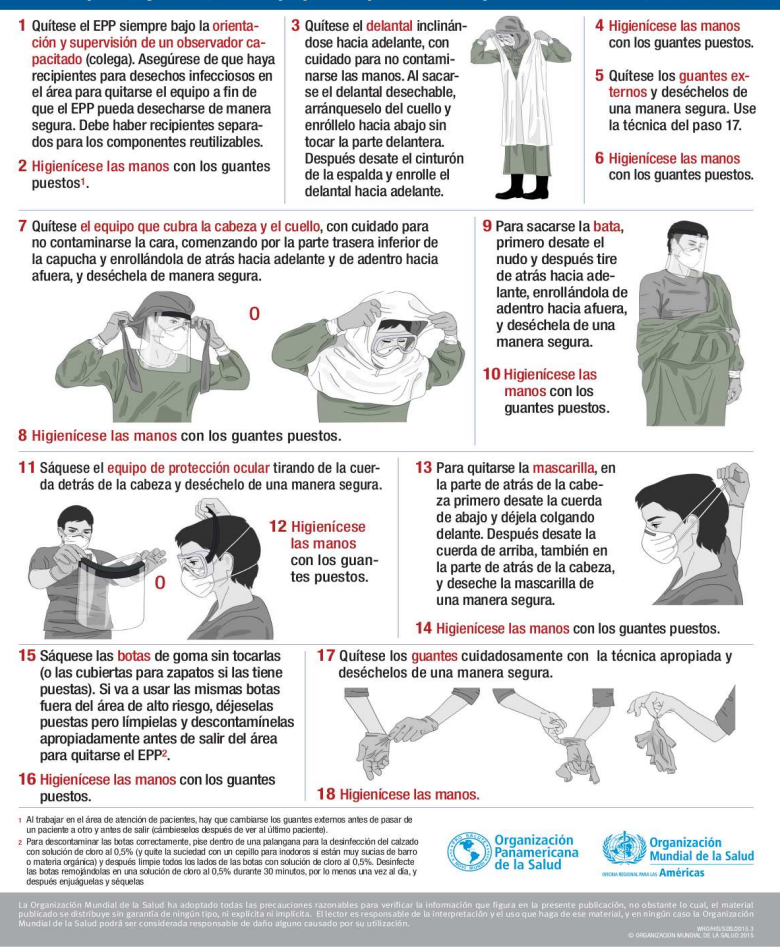

Figura 1: Información de la OMS sobre puesta y retirada del EPP

Procedimientos como los siguientes, pueden llegar a ser desarrollados con la ayuda de un robot diseñado a tal fin:

- Soporte para la comunicación con familiares

- Entrega de alimentos

- Consulta estado general, monitorización de constantes

- Desinfección de superficies

Hemos de tener en cuenta que cualquier robot cuya función esté relacionada con el cuidado de la salud ha de cumplir los siguientes requerimientos

\footnotetext{
$1_{\text {https://www.who.int/csr/resources/publications/PPE_EN_A1sl.pdf }}$
} 
[10], en base a ellos se pueden decidir las mejores políticas y estratégicas para mejorar la calidad de la atención médica :

- Cinemática y dinámica: Si se necesita que el robot tenga capacidad de manipular su entorno ha de tener múltiples múltiples grados de libertad (DOF), ser precisos y flexibles.

- Control y destreza: Ha de tener destreza suficiente para poder realizar todas las tareas asignadas de forma controlada, sin poner en peligro a los usuarios.

- Esterilización: Es necesario que el equipo disponga de mecanismos para poder esterilizar el entorno y así mismo.

- Seguridad: Debe disponer de mecanismos de seguridad.

- Requerimientos energéticos: El equipo debe poder controlar la carga disponible, volver a su base cuando requiera recarga y poder avisar de ello a su supervisor para que sea consciente del estado de la tarea encomendada.

- Coste: Es necesario pensar en un coste asumible por instituciones y naciones en desarrollo.

\section{Robots asistenciales en desarrollo}

Los robots asistenciales deberían cumplir con las siguientes funciones principales: Comunicación, movilidad, mediciones de datos clínicos, manipulación de su entorno y uso de herramientas.

Veamos algunos de ellos más detenidamente, en la siguiente tabla 1 se presenta un resumen de diferentes sistemas desarrollados, que cumplen en parte con las características anteriormente mencionadas.

\subsection{UVD Robot}

UVD Robot es un robot de desinfección (UVD Robots, Denmark), ver Figura 2, que tiene capacidad de localización y mapeo del entorno, la cual le permite desplazarse por si mismo de forma autónoma. UVD -Robots ${ }^{2}$, detecta la presencia de personas por medio de sensores para detenerse si está realizando la desinfección. Utiliza una tablet a modo de interfaz (incluida con el propio robot) o a través de una aplicación en el teléfono móvil. El robot debe ser configurado previamente

\footnotetext{
$2_{\text {https://www.uvd-robots.com/ }}$
}

Tabla 1: Sistemas robóticos asistenciales

\begin{tabular}{|c|c|c|c|}
\hline Dispositivo & Sensores & Multimedia & DOF \\
\hline Spot [4] & $\begin{array}{l}\text { Temp., RR } \\
\mathrm{HR}, \mathrm{SpO}_{2}\end{array}$ & LiDAR & - \\
\hline Aimbot [1] & $\begin{array}{l}\text { HD Infrared, Cámara } \\
\text { térmica, LiDAR }\end{array}$ & - & - \\
\hline Yumi [14] & $\begin{array}{c}\text { Doppler Ultrasound } \\
\text { Stethoscope }\end{array}$ & WebRTC & 14 Ejes \\
\hline ARI [5] & - & $\begin{array}{l}\text { NVIDIA Jetson } \\
\text { TX2, ReSpeaker } \\
\text { MicArray V2.0 }\end{array}$ & - \\
\hline TRINA [11] & $\begin{array}{l}\text { Temperatura } \\
\mathrm{SpO}_{2}\end{array}$ & $\begin{array}{c}\text { Cámara panorámica } \\
\qquad 180^{\circ}\end{array}$ & 26 Ejes \\
\hline XDBOT [9] & - & $\begin{array}{c}\text { LIDAR } \\
\text { Intel RealSenseF200 } \\
\text { 3D }\end{array}$ & 6 Ejes \\
\hline UVD [2] & Escáner Láser & Cámaras 3D & - \\
\hline $\begin{array}{c}\text { Sanbot } \\
\text { Elf robot [3] }\end{array}$ & $\begin{array}{l}\text { Temperatura } \\
\text { Movimiento }\end{array}$ & $\begin{array}{c}\text { Cámaras 3D } \\
\text { Full-HD touch }\end{array}$ & 2 Ejes \\
\hline Dispositivo & Desinfección & Batería & Coste \\
\hline Spot [4] & - & - & 74,500 \\
\hline Aimbot [1] & - & - & - \\
\hline Yumi [14] & $\checkmark$ & - & - \\
\hline ARI [5] & - & $8-12 \mathrm{~h}$ & - \\
\hline TRINA [11] & - & $24 \mathrm{~h}$ & $75,000 \$$ \\
\hline ХDBOT [9] & $\checkmark$ & $4 \mathrm{~h}$ & - \\
\hline UVD [2] & $\checkmark$ & - & $80,000 \$$ \\
\hline $\begin{array}{c}\text { Sanbot } \\
\text { Elf robot [3] }\end{array}$ & - & $4-10 \mathrm{~h}$ & $3,000 €$ \\
\hline
\end{tabular}

para memorizar todas las tareas que puede realizar y de esa forma ser autónomo.

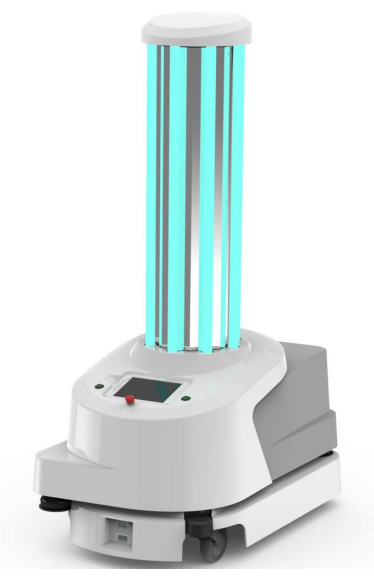

Figura 2: UVD ROBOTS https://www.uvdrobots.com/modelb 


\subsection{Xdbot}

Extreme Disinfection Robot, Nanyang Technological University, es un robot semi autónomo, con capacidad para explorar su entorno e identificar los objetos para ser desinfectados, posee además un brazo robótico controlado remotamente por un operador a través de un ordenador o tablet [9], ver figura 3

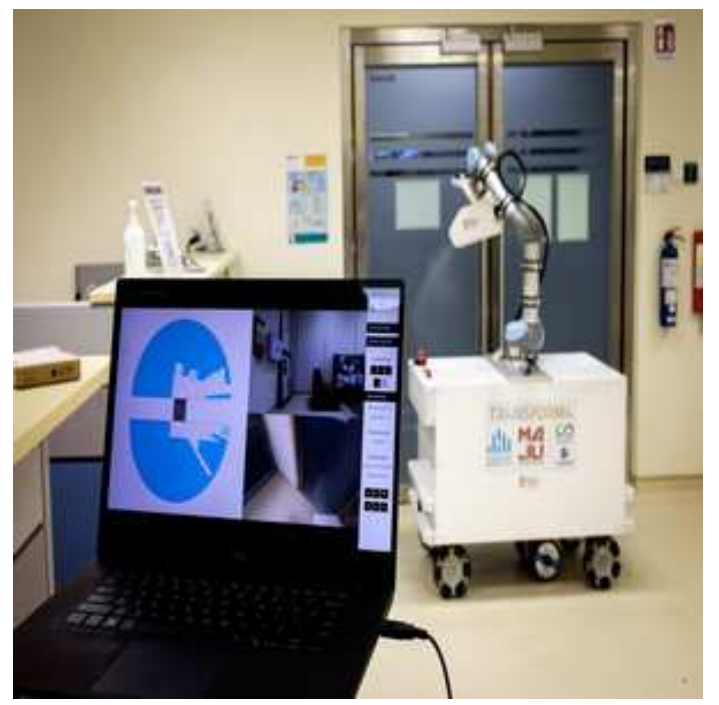

Figura 3: Interfaz XDBOT, COVID19; Chen IMing; Disinfection robot; RRC; Source:NTU Singapore

\subsection{TRINA}

El desarrollo del prototipo (TRINA) - TeleRobotic Intelligent Nursing Assistant de la Universidad de Illinois [11], ha surgido como respuesta a la necesidad de minimizar la exposición a posibles contagios. Para ello se ha diseñado un robot manipulador móvil, con una consola integrada para la que se ha diseñado un software específico, manipulada por un operador experto, puede realizar diferentes rutinas, como preparar y servir comida, transportar y entregar medicinas, también dispone de diversos sensores que permiten por ejemplo medir la temperatura y humedad de la habitación así como la saturación de oxígeno en la sangre $\mathrm{SpO}_{2}$ delpaciente.

El robot está compuesto por un torso humanoide "Rethink Robotics Baxter" [6], sobre una base omni direccional "HStar AMP-I" [13], y un sistema multimedia bidireccional. El dispositivo requiere estar conectado permanentemente a un cable de alimentación que le permite desplazarse por la habitación. Podemos ver los componentes en la figura 4

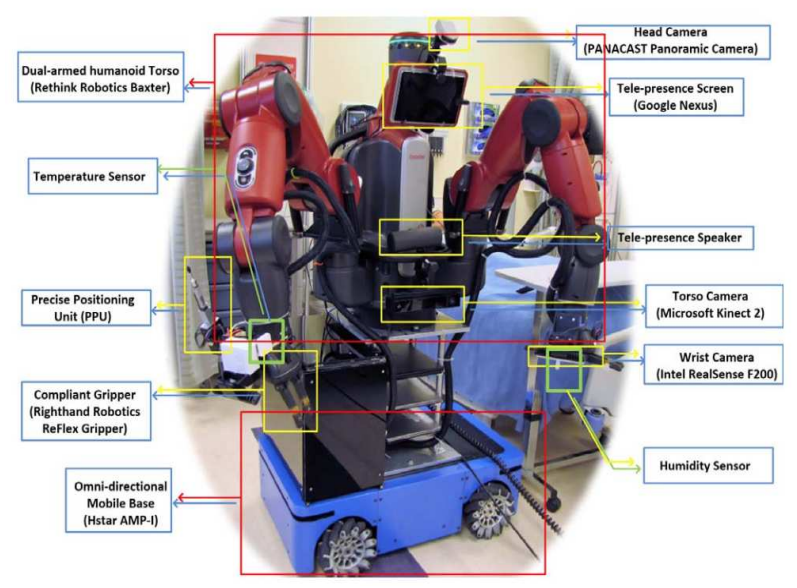

Figura 4: TRINA — the Tele-robotic Intelligent Nursing Assistant.

\subsection{Robot colaborativo teleoperado con doble brazo YuMi (ABB, Switzerland)}

El sistema telerobótico YuMi de ABB combina diferentes capacidades para trabajar en entornos peligrosos, así como la atención al paciente [14]. compuesto por dos subsistemas, un sistema teleoperado formado por un YuMi, IRB14000 [7], doble brazo que incluye manos flexibles, sistemas de alimentación de piezas, localización de piezas mediante cámaras y control robótico de última generación, a este subsistema se les une un sistema de telepresencia, compuesto por una tablet, con un sistema de comunicación basado en WebRTC [12].

El chasis se desplaza sobre "Mecanum wheels", lo que mejora la movilidad dentro de entornos aislados y reducidos, ya que podemos obtener desplazamientos en cualquier dirección mediante un control individual de la rotación de cada rueda y la combinación lineal de las fuerzas que esta genera [8].

\subsection{Entorno social}

Dentro de la robótica asistencial también existen iniciativas de diseñar robots con forma mecánica mas similar a un humano, como por ejemplo el Sanbot Elf robot (Qihan Technology, China) [3]. Como podemos ver en la figura 6 , se trata de un robot comercial, con una API y un código SDK, para poder integrar el Sanbot Elf en cualquier escenario. Se ha puesto especial cuidado en su apariencia humanoide, el reconocimiento por voz y al detección y reconocimiento facial. 


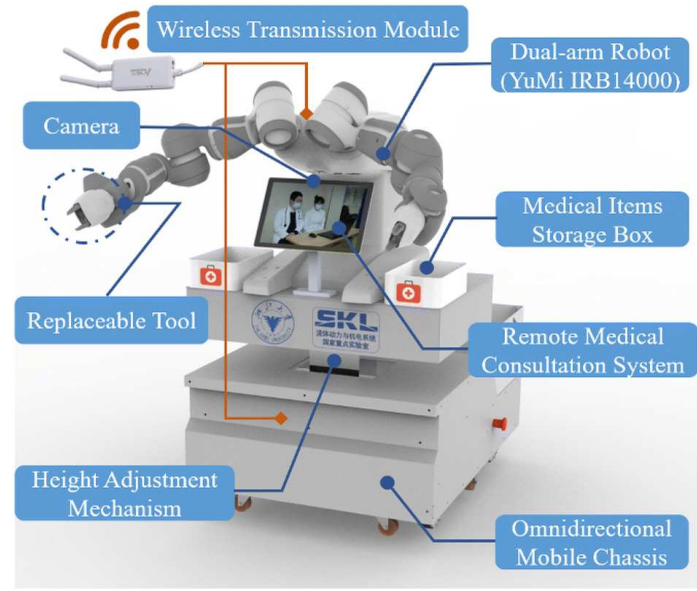

Figura 5: The teleoperation system consists of a wearable initial motion capture device and a dualarm collaborative robot (YuMi, IRB14000)..
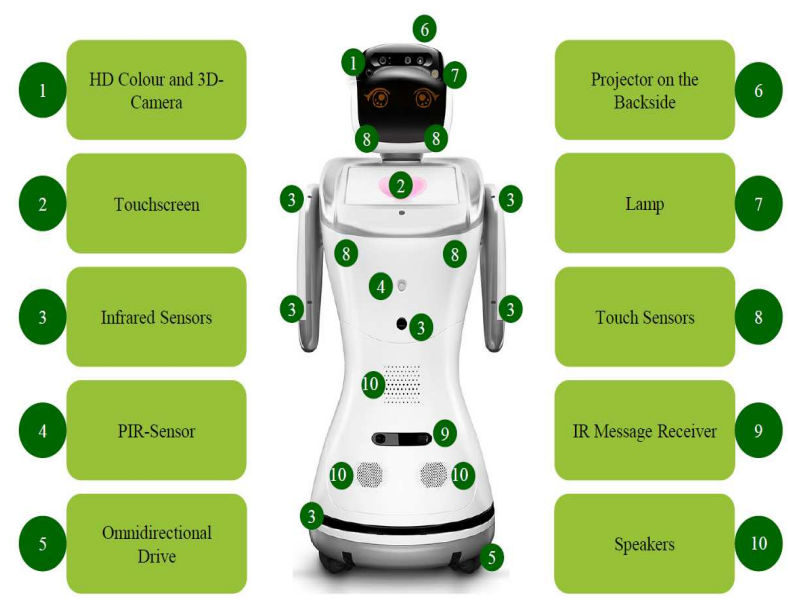

Figura 6: Hardware built into the Sanbot Elf. Picture of the Sanbot Elf: QIHAN Technology (2018a)

\section{Conclusiones y Trabajo Futuro}

En el presente artículo hemos examinado una selección de trabajos actuales en el ámbito de la robótica asistencial, especialmente en lo que respecta a la necesidad de apoyar a los técnicos sanitarios en escenarios de enfermedades contagiosas.

En varios de los ejemplos anteriores hemos visto que los dispositivos son muy pesados y voluminosos, como por ejemplo el robot TRINA [11], de $140 \mathrm{~kg}$ y una altura de $1.70 \mathrm{~cm}$, lo que limita la movilidad y agilidad del mismo. También, existe una tendencia a procurar sustituir las funciones de un sanitario, a través de un robot.

En nuestra opinión, siguiendo conversaciones con técnicos sanitarios, es bueno proponer un dispositivo de dimensiones reducidas, que pueda plegarse para poder ser transportado fácilmente, con un peso máximo de $20 \mathrm{~kg}$. El material ligero y el tamaño reducido nos permitirá reducir sensiblemente su coste de fabricación para poder tener varias unidades disponibles por departamento o área de trabajo. También, los técnicos sanitarios han manifestado la necesidad de dar una gran importancia al contacto personal de la persona confinada con un humano, en este caso el médico o el enfermero. Por ello, el sistema que nos han propuesto entra mas en el ámbito de la telerobótica supervisada, con grandes facilidades en la interfaz de usuario, concretamente para el de un usuario del ámbito sanitario que necesita interactuar con el robot no de forma manual, sino utilizando comandos de alto nivel (e.g. ir a la habitación del paciente, entregar comida, medir constantes, activar videoconferencia, etc.).

El trabajo futuro se centrará precisamente en avanzar en esta dirección, de la mano de los técnicos sanitarios, por medio de la realización de diferentes encuestas y entrevistas, podremos saber que necesidades debe cumplir el prototipo que vamos a desarrollar, procurando mejorar la seguridad y eficiencia, así como el desarrollo de una interfaz ágil y dinámica que pueda ser personalizada dependiendo de las necesidades del centro donde se va a distribuir el nuevo equipo, de forma que sea lo más eficiente posible para ayudar con la recuperación de las personas ante enfermedades contagiosas.

\section{Agradecimientos}

Este trabajo ha sido realizado por el equipo del CIRTESU, "Centro de Investigación en Robótica y Tecnologías Subacuáticas" de la Universidad Jaume I, y financiado por los proyectos DPI201786372-C3 (TWINBOT), IDIFEDER/2018/013 (GV), UJI-B2018-34 (NEPTUNO), y el proyecto H2020-Peacetolero-NFRP-2019-2020-04.

\section{English summary}

\section{AUTONOMOUS ROBOT \\ ASSISTANCE}

\begin{abstract}
In this article we show the state-of-the-art robotic systems that are under development (some of which are already in use), to operate in infectious environments, for example, risk areas associated with COVID-19. The systems allow healthcare professionals to work more safely, and also increase the safety of the patients themselves, allowing patient monitoring, as well as disinfection
\end{abstract}


of areas exposed to the virus, among other potential applications. Future work will show the results of interviews with healthcare personnel, who have presented requirements for a potential future robotic healthcare system in this field.

Keywords: COVID-19, Sanitary Robotics, Sanitation, Disinfection, Sensors.

\section{Referencias}

[1] Evan Ackerman. Why boston dynamics is putting legged robots in hospitals - ieee spectrum.

[2] Evan Ackerman. Autonomous robots are helping kill coronavirus in hospitals, 2020.

[3] Jochen Bauer, Lennarth Gruendel, Julian Sessner, Moritz Meiners, Markus Lieret, Tobias Lechler, Christoph Konrad, and Jörg Franke. 2 camera-based fall detection system with the service robot sanbot elf. 122018 .

[4] Amanda Bouman, Muhammad Fadhil Ginting, Nikhilesh Alatur, Matteo Palieri, David D. Fan, Thomas Touma, Torkom Pailevanian, Sung Kyun Kim, Kyohei Otsu, Joel Burdick, and Ali Akbar Agha-Mohammadi. Autonomous spot: Long-range autonomous exploration of extreme environments with legged locomotion. pages 2518-2525. Institute of Electrical and Electronics Engineers Inc., 102020.

[5] Sara Cooper, Alessandro Di Fava, Carlos Vivas, Luca Marchionni, and Francesco Ferro. Ari: The social assistive robot and companion. pages 745-751. Institute of Electrical and Electronics Engineers Inc., 82020.

[6] Sven Cremer, Lawrence Mastromoro, and Dan O. Popa. On the performance of the baxter research robot. pages 106-111. Institute of Electrical and Electronics Engineers Inc., 112016.

[7] Simon Duenser, James M. Bern, Roi Poranne, and Stelian Coros. Interactive robotic manipulation of elastic objects. pages $3476-$ 3481. Institute of Electrical and Electronics Engineers Inc., 122018.

[8] A. Gfrerrer. Geometry and kinematics of the mecanum wheel. Computer Aided Geometric Design, 25:784-791, 122008.
[9] Chen I-Ming. Researchers build disinfection robot to aid cleaners in covid-19 outbreak by nanyang technological university. 042020.

[10] Zeashan Hameed Khan, Afifa Siddique, and Chang Won Lee. Robotics utilization for healthcare digitization in global covid-19 management. International Journal of Environmental Research and Public Health, 17, 62020 .

[11] Zhi Li, Peter Moran, Qingyuan Dong, Ryan J. Shaw, and Kris Hauser. Development of a tele-nursing mobile manipulator for remote care-giving in quarantine areas. pages 3581-3586. Institute of Electrical and Electronics Engineers Inc., 72017.

[12] Paola Pierleoni, Luca Pernini, Lorenzo Palma, Alberto Belli, Simone Valenti, Lorenzo Maurizi, Loris Sabbatini, and Alessandro Marroni. An innovative webrtc solution for e-health services. Institute of Electrical and Electronics Engineers Inc., 11 2016.

[13] Eric C. Townsend, Erich A Mielke, David Wingate, and Marc D. Killpack. Estimating human intent for physical human-robot co-manipulation. 52017.

[14] Geng Yang, Honghao Lv, Zhiyu Zhang, Liu Yang, Jia Deng, Siqi You, Juan Du, and Huayong Yang. Keep healthcare workers safe: Application of teleoperated robot in isolation ward for covid-19 prevention and control. Chinese Journal of Mechanical Engineering (English Edition), 33:47, 122020.

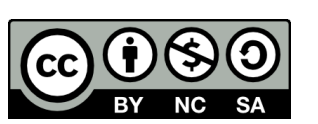
(C) 2021 by the authors. Submitted for possible open access publication under the terms and conditions of the Creative Commons Attribution CC BY-NC-SA 4.0 license (https://creativecommons.org/licenses/by-ncsa/4.0/deed.es). 